\title{
Retraction: An actin-dependent spindle position checkpoint ensures the asymmetric division in mouse oocytes
}

Aïcha Metchat, Manuel Eguren, Julius M. Hossain, Antonio Z. Politi, Sébastien Huet \& Jan Ellenberg

Nature Communications 6:7784 doi:10.1038/ncomms8784 (2015); Published 15 Jul 2015; Updated 29 Jul 2015

This paper has been retracted as it was submitted by the corresponding author A.M. without the knowledge or consent of the other researchers listed on the paper or their institution, utilising incorrect contact details. With the exception of the corresponding author, all other researchers have requested that the paper be retracted. 